INPLASY

PROTOCOL

To cite: Li et al. Computed tomography-guided lung biopsy: a meta-analysis of core needle and fine needle aspiration biopsy. Inplasy protocol 202110036. doi: 10.37766/inplasy2021.1.0036

Received: 12 January 2021

Published: 12 January 2021

Corresponding author: Ya-Yong Huang

yayonghuang@yeah.net

Author Affiliation:

Xuzhou Central Hospital

Support: None.

Review Stage at time of this submission: Preliminary searches.

Conflicts of interest: None.

\section{Computed tomography-guided lung biopsy: a meta-analysis of core needle and fine needle aspiration biopsy}

\author{
Li, Y1; Yang, F2; Huang, YY3; Fu, YF4.
}

Review question / Objective: Whether computed tomography (CT)-guided core needle biopsy (CNB) or fine-needle aspiration biopsy (FNAB) in lung disease is still controversial. This meta-analysis was conducted to provide evidence-based recommendations and to detect differences between CTguided CNB and FNAB in lung disease patients.

Condition being studied: Diagnostic accuracy can also be profoundly impacted by the type of needle selected for the biopsy procedure. Many studies to date have compared rates of sample adequacy, diagnostic accuracy, and complications between lung disease patients that underwent core needle biopsy (CNB) or fine-needle aspiration biopsy (FNAB). Several of these endpoints in these studies, however, were controversial, leading to inconsistent findings. For example, some studies found CT-guided CNB and FNAB to be associated with similar rates of diagnostic accuracy, whereas other studies found the diagnostic accuracy rate in patients that underwent CT-guided CNB to be significantly higher than that in patients that underwent CT-guided FNAB. In contrast, another study detected higher rates of diagnostic accuracy in the FNAB group. Studies with a large sample size will therefore be essential in order to accurately compare diagnostic accuracy rates and other endpoints between lung disease patients that underwent CT-guided CNB and FNAB.

INPLASY registration number: This protocol was registered with the International Platform of Registered Systematic Review and Meta-Analysis Protocols (INPLASY) on 12 January 2021 and was last updated on 12 January 2021 (registration number INPLASY202110036).

\section{INTRODUCTION}

Review question / Objective: Whether computed tomography (CT)-guided core needle biopsy (CNB) or fine-needle aspiration biopsy (FNAB) in lung disease is still controversial. This meta-analysis was conducted to provide evidence-based recommendations and to detect 
differences between CT-guided CNB and FNAB in lung disease patients.

Rationale: Sample adequacy, diagnostic accuracy, pneumothorax rates, hemorrhage rates, and rates of chest tube insertion for complication were pooled to compare the effectiveness of CNB and FNAB for lung lesions.

Condition being studied: Diagnostic accuracy can also be profoundly impacted by the type of needle selected for the biopsy procedure. Many studies to date have compared rates of sample adequacy, diagnostic accuracy, and complications between lung disease patients that underwent core needle biopsy (CNB) or fine-needle aspiration biopsy (FNAB). Several of these endpoints in these studies, however, were controversial, leading to inconsistent findings. For example, some studies found CT-guided CNB and FNAB to be associated with similar rates of diagnostic accuracy, whereas other studies found the diagnostic accuracy rate in patients that underwent CT-guided CNB to be significantly higher than that in patients that underwent CT-guided FNAB. In contrast, another study detected higher rates of diagnostic accuracy in the FNAB group. Studies with a large sample size will therefore be essential in order to accurately compare diagnostic accuracy rates and other endpoints between lung disease patients that underwent CT-guided CNB and FNAB.

\section{METHODS}

Search strategy: ((()(cutting[Title/Abstract]) OR (core[Title/Abstract])) OR (biopsy[Title/ Abstract])) AND ((fine needle[Title) Abstract]) OR (aspiration[Title/Abstract]))) AND ((computed tomography[Title) Abstract]) OR (CT[Title/Abstract]))) AND (((lung[Title/Abstract]) OR (pulmonary[Title/ Abstract])) OR (thoracic[Title/Abstract])).

Participant or population: Patients with lung lesions who underwent CT guided CNB or FNAB.

Intervention: CT-guided CNB procedures.
Comparator: CT-guided FNAB procedures.

Study designs to be included: Studies were eligible for inclusion if they met the following criteria: (a) studies were either randomized controlled trials (RCTs) or nonrandomized studies comparing CT-guided CNB and FNAB analyses of lung disease patients; and (b) studies were published in English. Studies were excluded if they were: (a) non-comparative studies; (b) animal studies; or (c) reviews.

Eligibility criteria: Studies were eligible for inclusion if they met the following criteria: (a) studies were either randomized controlled trials (RCTs) or non-randomized studies comparing CT-guided CNB and FNAB analyses of lung disease patients; and (b) studies were published in English. Studies were excluded if they were: (a) noncomparative studies; (b) animal studies; or (c) reviews.

Information sources: We searched the Pubmed, Embase, and Cochrane Library databases for relevant studies published as of June 2020.

Main outcome(s): Diagnostic accuracy.

Additional outcome(s): Sample adequacy, pneumothorax rates, hemorrhage rates, and rates of chest tube insertion for complication.

Data management: RevMan version $\mathbf{5 . 3}$ was used for all meta-analyses. Dichotomous variables were analyzed via the Mantel-Haenszel method to assess pooled odds ratios (ORs) and $95 \%$ confidence intervals (Cls). Heterogeneity was analyzed based upon $\mathrm{X} 2$ tests and the 12 statistic, with $12>50 \%$ being indicative of significant heterogeneity. When significant heterogeneity was found, metaanalyses were performed with a randomeffects model, whereas they were otherwise analyzed by a fixed-effects model. Potential sources of heterogeneity were evaluated through subgroup and sensitivity analyses, while the publication bias was assessed by funnel plots. 
Quality assessment / Risk of bias analysis: The Jadad composite scale was used to assess the quality of all RCTs, while the retrospective study's quality was assessed using the Newcastle-Ottawa scale, with a maximum possible quality score of 9 points.

Strategy of data synthesis: Two researchers independently extracted all data from included studies, with a third researcher resolving any discrepancies. Extracted data included baseline study parameters, patient baseline data, and biopsy-associated outcomes.

Subgroup analysis: Subgroup analysis is performed based on lung nodules.

Sensibility analysis: None.

Language: English.

Country(ies) involved: China.

Other relevant information: None.

Keywords: Lung; Biopsy; Core needle; Fine needle.

Contributions of each author:

Author 1 - Yong Li - The author drafted the manuscript.

Author 2 - Fang Yang - The author provided statistical expertise.

Author 3 - Ya-Yong Huang - The author contributed to the development of the selection criteria, and the risk of bias assessment strategy.

Author 4 - Yu-Fei Fu - The author read, provided feedback and approved the final manuscript. 